\title{
Maximal Element Theorems In GFC-Spaces With The Application To Systems of General Quasiequilibrium Problems
}

\author{
Kai Ting Wen \\ Library of Guizhou University of Engineering Science, Bijie, Guizhou 551700, P. R. China \\ Wenkaiting_2004@sina.com
}

\begin{abstract}
Keywords: GFC-space, GFS-KKM mapping, $G_{K S}$-majorized mapping, Maximal element, System of general quasiequilibrium problems, Equilibrium.

Abstract. $G_{K S}$-mappings and $G_{K S}$-majorized mappings are introduced, and maximal element theorems for $G_{K S}$-mappings and $G_{K S}$-majorized mappings are established in GFC-spaces. As application, a new equilibrium existence theorem for systems of general quasiequilibrium problems is obtained.
\end{abstract}

\section{Introduction}

In 2009, Khanh et al.[1, 2] introduced GFC-spaces. In 2010, Khanh et al.[3] established coincidence theorems, maximal elements and nonempty intersections in GFC-spaces. In 2011, Wen[4,5] established intersection theorems, fixed point theorems, variational inequalities and existence theorems for solutions of generalized equilibrium problems with lower and upper bounds in GFC-spaces. In 2012, Wen[6] obtained equilibrium existence theorems for systems of quasiequilibrium problems and systems of general quasiequilibrium problems in GFC-spaces. In 2013, Wen et al.[7-9] obtained matching theorems, saddle point theorems, section theorems and existence theorems of solutions for systems of generalized mixed vector quasiequilibrium problems in GFC-spaces. In 2014, Wen et al.[10-11] established equilibrium existence theorems for systems of general quasiequilibrium problems and abstract economies in GFC-spaces. In 2015, Wen et al.[12-15] studied equilibrium existence for constrained multiobjective games, abstract generalized vector equilibrium problems, coincidence problems and GFC-KKM theorem in GFC-spaces.

In this paper, $G_{K S}$-mappings and $G_{K S}$-majorized mappings are introduced, and maximal element theorems for $G_{K S}$-mappings and $G_{K S}$-majorized mappings are established in GFC-spaces. As application, a new equilibrium existence theorem for systems of general quasiequilibrium problems is obtained.

\section{Preliminaries}

Let $X$ be a nonempty set. We denote by $<X>$ and $2^{X}$ the family of all nonempty finite subsets of $X$ and the family of all subsets of $X$, respectively, by $\triangle_{n}$ the standard $n$-dimensional simplex with vertices $e_{0}, \ldots, e_{n}$. Let $X$ and $Y$ be topological spaces. We denote by $C(X, Y)$ the class of single-valued continuous maps of $X$ into $Y$. Following Khanh et al.[1-15], let $X$ be a topological space, $Y$ be a nonempty set and $\Phi$ a family of continuous mappings $\varphi_{N}: \Delta_{n} \rightarrow X .(X, Y, \Phi)$ is said to be a GFC-space if for each $N:=\left\{y_{0}, \cdots, y_{n}\right\} \in\langle Y\rangle$, there exists $\varphi_{N}: \Delta_{n} \rightarrow X$ of the family $\Phi$. Let $(X, Y, \Phi)$ be a GFC-space, $Z$ a nonempty set, $S: X \rightarrow Z$ a map. $T: Y \rightarrow 2^{Z}$ a mapping. $T$ is said to be a GFS-KKM mapping if for each $N:=\left\{y_{0}, \cdots, y_{n}\right\} \in\langle Y\rangle,\left\{y_{i_{0}}, \cdots, y_{i_{k}}\right\} \in\langle N\rangle$, we have $S\left(\varphi_{N}\left(\Delta_{k}\right)\right) \subset \bigcup_{j=0}^{k} T\left(y_{i_{j}}\right)$.

Now, we introduce the following definitions.

\section{Definition 2.1}

Let $(X, Y, \Phi)$ be a GFC-space, $Z$ a topological space, $K$ a nonempty compact subset of $Z$. $S: X \rightarrow Z$ a map. $T: Z \rightarrow 2^{Y}$ is said to be a $G_{K S}$-mapping if $T$ has weakly compactly local intersection property 
relatively to $K$ and for each $N:=\left\{y_{0}, \cdots, y_{n}\right\} \in\langle Y\rangle,\left\{y_{i_{0}}, \cdots, y_{i_{k}}\right\} \in\langle N\rangle$, we have $S\left(\varphi_{N}\left(\Delta_{k}\right)\right) \cap \bigcap_{j=0}^{k} c \operatorname{int}_{Z} T^{-1}\left(y_{i_{j}}\right)=\varnothing$.

\section{Definition 2.2}

Let $(X, Y, \Phi)$ be a GFC-space, $Z$ a topological space, $K$ a nonempty compact subset of $Z$. $S: X \rightarrow Z$ a map. $T: Z \rightarrow 2^{Y}$ is said to be a $G_{K S}$-majorized mapping if for each $z \in Z$ satisfying $T(z) \neq \varnothing$, there exists a mapping $T_{z}: Z \rightarrow 2^{Y}$ and an open neighborhood $N(z) \subset Z$ of $z$ in $Z$ such that

(1) $T_{z}^{-1}$ is weakly transfer compactly open valued relatively to $K$;

(2) $T(x) \subset T_{z}(x)$ for each $x \in N(z)$;

(3) for each $N:=\left\{y_{0}, \cdots, y_{n}\right\} \in\langle Y\rangle,\left\{y_{i_{0}}, \cdots, y_{i_{k}}\right\} \in\langle N\rangle$, we have

$$
N(z) \cap S\left(\varphi_{N}\left(\Delta_{k}\right)\right) \cap \bigcap_{j=0}^{k} \operatorname{cint}_{z} T_{z}^{-1}\left(y_{i_{j}}\right)=\varnothing .
$$

\section{Remark 2.1}

Definition 2.1 and 2.2 unify and generalize correlated definitions in Wu[16], Wen[17], Wen[18], Yang[19] and Ding[20].

The following lemma is Theorem 3.1 of Wen[15].

\section{Lemma 2.1}

Let $(X, Y, \Phi)$ be a GFC-space, $Z$ a topological space, $S \in O(X, Z)$ a continuous map, $F: Y \rightarrow 2^{Z}$ a GFS-KKM mapping with compactly closed values. Then

(1) $\{F(y)\}_{y \in Y}$ has the finite intersection property;

(2) $\bigcap_{y \in Y} F(y) \neq \varnothing$ if there exists a nonempty compact subset $K \subset Z$ and $M \in\langle Y\rangle$ such that $\bigcap_{y \in M} F(y) \subset K$.

\section{Main Results}

\section{Theorem 3.1}

Let $(X, Y, \Phi)$ be a GFC-space, $Z$ a topological space, $K$ a nonempty compact subset of $Z$. $S \in O(X, Z)$ a continuous map, $T: Z \rightarrow 2^{Y}$ a $G_{K S}$-mapping. Suppose that there exists $M \in\langle Y\rangle$ such that $Z \backslash K \subset \bigcup_{y \in M} \operatorname{cint}_{Z} T^{-1}(y)$. Then there exists $\hat{z} \in K$ such that $T(\hat{z})=\varnothing$.

Proof Define $F: Y \rightarrow 2^{Z}$ by $F(y):=Z \backslash c$ int $_{Z} T^{-1}(y)$ for each $y \in Y$. Since $c$ int $_{Z} T^{-1}(y)$ is compactly open for each $y \in Y$, then $F$ is compactly closed valued. Note that $T$ is a $G_{K S}$-mapping. Then for each $N:=\left\{y_{0}, \cdots, y_{n}\right\} \in\langle Y\rangle,\left\{y_{i_{0}}, \cdots, y_{i_{k}}\right\} \in\langle N\rangle$, we have $S\left(\varphi_{N}\left(\Delta_{k}\right)\right) \cap \bigcap_{j=0}^{k} c \operatorname{int}_{Z} T^{-1}\left(y_{i_{j}}\right)=\varnothing$, i.e.,

$$
S\left(\varphi_{N}\left(\Delta_{k}\right)\right) \subset Z \backslash \bigcap_{j=0}^{k} \operatorname{cint}_{Z} T^{-1}\left(y_{i_{j}}\right)=\bigcup_{j=0}^{k}\left(Z \backslash \operatorname{cint}_{Z} T^{-1}\left(y_{i_{j}}\right)\right)=\bigcup_{j=0}^{k} F\left(y_{i_{j}}\right),
$$

which means that $F$ is a GFS-KKM mapping with compactly closed values. Since there exists $M \in\langle Y\rangle$ such that $Z \backslash K \subset \bigcup_{y \in M} c \operatorname{int}_{Z} T^{-1}(y)$, then

$$
\bigcap_{y \in M} F(y)=\bigcap_{y \in M}\left(Z \backslash \operatorname{cint}_{Z} T^{-1}\left(y_{i_{j}}\right)\right)=Z \backslash \bigcup_{y \in M} \operatorname{cint}_{Z} T^{-1}\left(y_{i_{j}}\right) \subset K .
$$

By lemma 2.1, we have $\bigcap_{y \in Y} F(y) \neq \varnothing$. Take $\hat{z} \in \bigcap_{y \in Y} F(y) \subset \bigcap_{y \in M} F(y) \subset K$, we claim that $T(\hat{z})=\varnothing$. Otherwise, since $T$ is $G_{K S}$-mapping, then $T$ has weakly compactly local intersection property relatively to $K$. Define $T_{K}: K \rightarrow 2^{Y}$ by $T_{K}(z)=T(z)$ for each $z \in K$. Since $K$ is compact, than $T_{K}$ has local intersection property. Note that $\hat{z} \in K$ and $T_{K}(\hat{z})=T(\hat{z}) \neq \varnothing$. Hence, there exists an open neighborhood $N(\hat{z}) \subset Z$ such that $\bigcap_{z \in N(\hat{z})} T_{K}(z) \neq \varnothing$. Take $\hat{y} \in \bigcap_{z \in N(\hat{z})} T_{K}(z)$. Then $N(\hat{z}) \subset T_{K}^{-1}(\hat{y})$, so 
that $\hat{z} \in \operatorname{int}_{K} T_{K}^{-1}(\hat{y})=c \operatorname{int}_{K} T_{K}^{-1}(\hat{y}) \subset c \operatorname{int}_{Z} T_{K}^{-1}(\hat{y})$, and hence $\hat{z} \notin Z \backslash c \operatorname{int}_{K} T_{K}^{-1}(\hat{y})=F(\hat{y}), \quad$ a contradiction to $\hat{z} \in \bigcap_{y \in Y} F(y)$.

\section{Remark 3.1}

Theorem 3.1 unifies, improves and generalizes Theorem 1 of $\mathrm{Wu}[16]$, Theorem 1 of Wen[17], Theorem 2.1 of Wen[18].

\section{Theorem 3.2}

Let $(X, Y, \Phi)$ be a GFC-space, $Z$ a Hausdorff topological space, $K$ a compact subspace of $Z$. $S \in O(X, Z)$ a continuous map, $F: Z \rightarrow 2^{Y}$ a $G_{K S}$-majorized mapping. Then there exists $\hat{z} \in K$ such that $F(\hat{z})=\varnothing$. Proof Suppose that the conclusion is false, i.e.,

(1) $F(z) \neq \varnothing$ for each $z \in K$.

Since $F$ is a $G_{K S}$-majorized mapping, then for each $z \in K$, there exists a mapping $F_{Z}: Z \rightarrow 2^{Y}$ and an open neighborhood $N(z) \subset Z$ of $z$ in $Z$ such that

(2) $F_{z}^{-1}$ is weakly transfer compactly open valued relatively to $K$;

(3) $F(x) \subset F_{Z}(x)$ for each $x \in N(z)$;

(4) for each $N:=\left\{y_{0}, \cdots, y_{n}\right\} \in\langle Y\rangle,\left\{y_{i_{0}}, \cdots, y_{i_{k}}\right\} \in\langle N\rangle$, we have

$$
N(z) \cap S\left(\varphi_{N}\left(\Delta_{k}\right)\right) \cap \bigcap_{j=0}^{k} \operatorname{cint}_{Z} F_{z}^{-1}\left(y_{i_{j}}\right)=\varnothing .
$$

Define $T: K \rightarrow 2^{Y}$ by $T(z)=F(z)$ and $T_{Z}: K \rightarrow 2^{Y}$ by $T_{Z}(z)=F_{Z}(z)$ for each $z \in K$. Since $K$ is compact, then in virtue of (2), we have

(5) $T_{z}^{-1}$ is transfer open valued for each $z \in K$.

For each $z \in K$, we may suppose that open neighborhood $N(z)$ is also an open neighborhood of $z$ in $K$. Then by (3), we have

(6) $T(x) \subset T_{Z}(x)$ for each $x \in N(z)$

Note that $K$ is compact. Hence for each $y \in Y$, we have

$$
\operatorname{int}_{K} T_{Z}^{-1}(y)=\operatorname{cint}_{K} T_{Z}^{-1}(y) \subset \operatorname{cint}_{Z} T_{z}^{-1}(y) \subset \operatorname{cint}_{Z} F_{z}^{-1}(y) .
$$

Hence, (4) implies that

(7) for each $z \in K, N:=\left\{y_{0}, \cdots, y_{n}\right\} \in\langle Y\rangle,\left\{y_{i_{0}}, \cdots, y_{i_{k}}\right\} \in\langle N\rangle$, we have

$$
N(z) \cap S\left(\varphi_{N}\left(\Delta_{k}\right)\right) \cap \bigcap_{j=0}^{k} \operatorname{cint}_{Z} T_{z}^{-1}\left(y_{i_{j}}\right)=\varnothing .
$$

Since $Z$ is a Hausdorff topological space and $K$ is a compact subspace of $Z$, then $K$ is a compact Hausdorff topological space, and hence $K$ is a regular topological space, so that for each $z \in K$ and the open neighborhood $N(z)$ of $z$ in $K$, there exists an open neighborhood $U(z)$ of $z$ in $K$ such that $U(z) \subset c l_{K} U(z) \subset N(z)$. By the compactness of $K$, we have

(8) there exists $\left\{z_{0}, \cdots, z_{m}\right\} \in\langle K\rangle$ such that $K \subset \bigcup_{i=0}^{m} U\left(z_{i}\right)$.

For each $i \in\{0, \cdots, m\}$, define $T_{i}: K \rightarrow 2^{Y}$ by

$$
T_{i}(z):= \begin{cases}T_{z_{i}}(z), & \text { if } \quad z \in c l_{K} U\left(z_{i}\right), \\ Y, & \text { if } \quad z \notin c l_{K} U\left(z_{i}\right) .\end{cases}
$$

Then $T_{i}^{-1}(y)=T_{z_{i}}^{-1}(y) \cup\left(K \backslash c l_{K} U\left(z_{i}\right)\right)$ for each $y \in Y$. By (5), for each $i \in\{0, \cdots, m\}, T_{z_{i}}^{-1}$ is transfer open valued. Note that $K \backslash c l_{K} U\left(z_{i}\right)$ is open. Hence,

(9) for each $i \in\{0, \cdots, m\}, T_{i}^{-1}$ is transfer open valued.

Define $\tilde{T}: K \rightarrow 2^{Y}$ by $\tilde{T}(z):=\bigcap_{i=0}^{m} T_{i}(z)$ for each $z \in Z$. We claim that $\tilde{T}$ is a $G_{K S}$-mapping. First of all, since $\tilde{T}^{-1}(y):=\bigcap_{i=0}^{m} T_{i}^{-1}(y)$ for each $y \in Y$, then $\tilde{T}^{-1}$ is transfer open valued by (9). In virtue of Lemma 1.3 of Wen[21], $\tilde{T}$ has local intersection property, so that $\tilde{T}$ has weakly compactly local 
intersection property relatively to $K$. Secondly, for each $N:=\left\{y_{0}, \cdots, y_{n}\right\} \in\langle Y\rangle,\left\{y_{i_{0}}, \cdots, y_{i_{k}}\right\} \in\langle N\rangle$, we claim that $S\left(\varphi_{N}\left(\Delta_{k}\right)\right) \cap \bigcap_{j=0}^{k} \operatorname{cint}_{K} \tilde{T}^{-1}\left(y_{i_{j}}\right)=S\left(\varphi_{N}\left(\Delta_{k}\right)\right) \cap \bigcap_{j=0}^{k} \operatorname{int}_{K} \tilde{T}^{-1}\left(y_{i_{j}}\right)=\varnothing$. Otherwise, there exist $N:=\left\{y_{0}, \cdots, y_{n}\right\} \in\langle Y\rangle,\left\{y_{i_{0}}, \cdots, y_{i_{k}}\right\} \in\langle N\rangle$ such that $S\left(\varphi_{N}\left(\Delta_{k}\right)\right) \cap \bigcap_{j=0}^{k} \operatorname{int}_{K} \tilde{T}^{-1}\left(y_{i_{j}}\right) \neq \varnothing$. Take $z^{*} \in S\left(\varphi_{N}\left(\Delta_{k}\right)\right) \cap \bigcap_{j=0}^{k} \operatorname{int}_{K} \tilde{T}^{-1}\left(y_{i_{j}}\right) \subset K$. Then by (8), there exists $p \in\{0, \cdots, m\}$ such that $z^{*} \in U\left(z_{p}\right) \subset N\left(z_{p}\right)$. Note that

$$
z^{*} \in \bigcap_{j=0}^{k} \operatorname{int}_{K} \tilde{T}^{-1}\left(y_{i_{j}}\right)=\bigcap_{j=0}^{k} \operatorname{int}_{K}\left(\bigcap_{i=0}^{m} T_{i}^{-1}\left(y_{i_{j}}\right)\right) \subset \bigcap_{j=0}^{k} \operatorname{int}_{K} T_{p}^{-1}\left(y_{i_{j}}\right) .
$$

Hence, there exists an open neighborhood $O\left(z^{*}\right)$ of $z^{*}$ in $K$ such that $O\left(z^{*}\right) \subset U\left(z_{p}\right) \cap \bigcap_{j=0}^{k} T_{p}^{-1}\left(y_{i_{j}}\right)$. For each $z \in O\left(z^{*}\right) \subset U\left(z_{p}\right) \cap \bigcap_{j=0}^{k} T_{p}^{-1}\left(y_{i_{j}}\right)$, by the definition of $T_{i}$, we have $\left\{y_{i_{0}}, \cdots, y_{i_{k}}\right\} \subset T_{p}(z)$ $=T_{z_{p}}(z)$, which implies that $z \in \bigcap_{j=0}^{k} T_{z_{p}}^{-1}\left(y_{i_{j}}\right)$, and then $O\left(z^{*}\right) \subset \bigcap_{j=0}^{k} T_{z_{p}}^{-1}\left(y_{i_{j}}\right)$, i.e., $z^{*} \in$ $\bigcap_{j=0}^{k}$ int $_{K} T_{z_{p}}^{-1}\left(y_{i_{j}}\right)$. So we have $z^{*} \in N\left(z_{p}\right) \cap S\left(\varphi_{N}\left(\Delta_{k}\right)\right) \cap \bigcap_{j=0}^{k} \operatorname{int}_{K} T_{z_{p}}^{-1}\left(y_{i_{j}}\right)$, which is a contradiction to (7). Finally, since $\tilde{T}$ is a $G_{K S}$-mapping and $K$ is compact, then for each $M \in\langle Y\rangle$, we have $K \backslash K \subset \bigcup_{y \in M} c \operatorname{int}_{z} \tilde{T}^{-1}(y)$. In virtue of Theorem 3.1, there exists $\hat{z} \in K$ such that $\tilde{T}(\hat{z})=\varnothing$. By (6) and the condition of $T_{i}$, for each $z \in K, i \in\{0, \cdots, m\}, F(z)=T(z) \subset T_{i}(z)$, hence, $F(z) \subset \bigcap_{i=0}^{m} T_{i}(z)=\tilde{T}(z)$, especially, $F(\hat{z}) \subset \tilde{T}(\hat{z})=\varnothing$, which is a contradiction to (1).

\section{Remark 3.1}

Theorem 3.2 unifies, improves and generalizes Theorem 2 of $\mathrm{Wu}[16]$, Theorem 2 of Wen[17], Theorem 2.2 of Wen[18].

\section{Theorem 3.3}

Let $I$ be a finite or infinite index set, for each $i \in I,\left(X_{i}, Y_{i}, \Phi_{i}\right)$ be a GFC-space, $Z_{i}$ a Hausdorff topological space, $Y_{i} \subset Z_{i}, T_{i}: Z:=\prod_{j \in I} Z_{j} \rightarrow Y_{i}$ a map, $A_{i}: Z \rightarrow 2^{Y_{i}} \backslash\{\varnothing\}$ a nonempty valued mapping, $\psi_{i}: Z \times Y_{i} \times Z \rightarrow \bar{R}$ a function, $K$ a compact subspace of $Z, X:=\prod_{j \in I} X_{j}, Y:=\prod_{j \in I} Y_{j}, S \in O(X, Z)$ a continuous map. Suppose that $A: Z \rightarrow 2^{Y}$ defined by $A(z):=\prod_{i \in I} A_{i}(z)$ for each $z \in Z$ is a $G_{K S}$-majorized mapping. Then the system of general quasiequilibrium problems $\operatorname{SGQEP}\left(T_{i}, A_{i}, \psi_{i}\right)_{i \in I}$ has an equilibrium in $Z$, i.e., there exists $\hat{z} \in Z$ such that

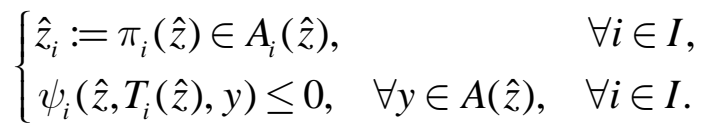

Proof For each $i \in I, N_{i} \in\left\langle Y_{i}\right\rangle, \varphi_{N_{i}} \in \Phi_{i}$, let $\Phi=\left\{\varphi_{N}: \varphi_{N}:=\prod_{i \in I} \varphi_{N_{i}}\right\}$. Then $(X, Y, \Phi)$ is a GFC-space. Since $Z_{i}$ is a Hausdorff topological space for each $i \in I$, then $Z:=\prod_{j \in I} Z_{i}$ is a Hausdorff topological space. For each $i \in I$, define $P_{i}: Z \rightarrow 2^{Y}$ by $P_{i}(z):=\left\{y \in Y: \psi_{i}\left(z, T_{i}(z), y\right)>0\right\}$ for each $z \in Z$. Let $D:=\{z \in Z: z \in A(z)\}$. Define $F: Z \rightarrow 2^{Y}$ by

$$
F(z):= \begin{cases}A(z), & \text { if } z \in Z \backslash D, \\ A(z) \cap \bigcup_{i \in I} P_{i}(z), & \text { if } z \in D .\end{cases}
$$

We claim that there exists $\hat{z} \in Z$ such that $F(\hat{z})=\varnothing$. Otherwise, since $A$ is a $G_{K S}$-majorized mapping, then for each $z \in Z$, there exists $A_{z}: Z \rightarrow 2^{Y}$ and an open neighborhood $N(z) \subset Z$ such that

(1) $A_{z}^{-1}$ is weakly transfer compactly open valued relatively to $K$; 
(2) $A(x) \subset A_{z}(x)$ for each $x \in N(z)$;

(3) for each $N:=\left\{y_{0}, \cdots, y_{n}\right\} \in\langle Y\rangle,\left\{y_{i_{0}}, \cdots, y_{i_{k}}\right\} \in\langle N\rangle$, we have

$$
N(z) \cap S\left(\varphi_{N}\left(\Delta_{k}\right)\right) \cap \bigcap_{j=0}^{k} \operatorname{cint}_{Z} A_{z}^{-1}\left(y_{i_{j}}\right)=\varnothing .
$$

By the definition of $F, F(z) \subset A(z)$ for each $z \in Z$, hence by (2) we have

(4) $F(x) \subset A_{z}(x)$ for each $x \in N(z)$.

By (1), (3) and (4), $F$ is a $G_{K S}$-majorized mapping. In virtue of Theorem 3.2, there exists $\tilde{z} \in K \subset Z$ such that $F(\tilde{z})=\varnothing$, which is a contradiction. Finally, since $A_{i}$ is nonempty valued, then $A$ is nonempty valued. Note that $F(\hat{z})=\varnothing$. By the definition of $F$, we have $\hat{z} \in D$ and $A(\hat{z}) \cap \bigcup_{i \in I} P_{i}(\hat{z})=\varnothing$. Since $\hat{z} \in D$, hence $\hat{z} \in A(\hat{z})$, and then $\hat{z}_{i}:=\pi_{i}(\hat{z}) \in A_{i}(\hat{z})$ for each $i \in I$. Since $A(\hat{z}) \cap \bigcup_{i \in I} P_{i}(\hat{z})=\varnothing$, which implies that for each $y \in A(\hat{z}), y \notin \bigcup_{i \in I} P_{i}(\hat{z})$, and then for each $i \in I, y \notin P_{i}(\hat{z})$, i.e., for each $i \in I, \quad y \in A(\hat{z}), \psi_{i}\left(\hat{z}, T_{i} \hat{z}, y\right) \leq 0$. Therefore, $\hat{z} \in Z$ is an equilibrium of the system of general quasiequilibrium problems $\operatorname{SGQEP}\left(T_{i}, A_{i}, \psi_{i}\right)_{i \in I}$ in $Z$.

\section{Acknowledgements}

This work was financially supported by Natural Science Foundation of China (11361003) and the Natural Scientific Research Foundation of Guizhou Provincial Education Department((2012)058).

\section{References}

[1] P. Q. Khanh, N. H. Quan and J. C. Yao, Nonlinear Anal., Vol. 71(2009), p. 1227.

[2] N. X. Hai, P. Q. Khanh and N. H. Quan, Nonlinear Anal., Vol. 71(2009), p. 6170.

[3] P. Q. Khanh, N. H. Quan, Quan, Nonlinear Anal., Vol. 72(2010), p. 2706.

[4] K. T. Wen, $2^{\text {nd }}$ International Conference on Artificial Intelligence, Management Science and Electronic Commerce, Vol. 2(2011), p. 923.

[5] K. T. Wen, H. R. Li, 2011 World Congress on Engineering and Technology, Vol. 1(2011), p. 543.

[6] K. T. Wen, Acta Anal. Funct. Appl. Vol. 14(3)(2012), p. 292.

[7] K. T. Wen, H.R. Li, Advanced Materials Research, Vol. 734-737(2013), p. 2867.

[8] K. T. Wen, Applied Mechanics and Materials, Vol. 405-408(2013), p. 3151.

[9] K. T. Wen, J. Bijie University, Vol. 31(4)(2013) , p. 62.

[10] K. T. Wen, H.R. Li, Applied Mechanics and Materials, Vol. 556-562(2014), p. 4128.

[11] K. T. Wen, H.R. Li, Applied Mechanics and Materials, Vol. 587-589(2014), p. 2279.

[12] K. T. Wen, H.R. Li, Advanced Materials Research, Vol. 1065-1069(2015), p. 3450.

[13] H.R. Li, K. T. Wen, J. Guizhou University of Engineering Scinence, Vol. 33(3)(2015), p. 139.

[14] K. T. Wen, H.R. Li, J. Sichuan Normal University(Natural Science), Vol. 38(3)(2015), p. 368.

[15] K. T. Wen, Math. Appl., Vol. 28(3)(2015), p. 533.

[16] X. Wu, Comput. Math. Appl., Vol. 40(2000), p. 1097.

[17] K. T. Wen, Math. Appl. China, Vol. 21(2)(2008), p. 239.

[18] K. T. Wen, Adv. Math. China, Vol. 42(4)(2013), p. 527. 
[19] M. G. Yang, Nonlinear Anal., Vol. 70(2009), p. 890.

[20] X. P. Ding, Nonlinear Anal., Vol. 72(2010), p. 65.

[21]K. T. Wen, Adv. Math. China, Vol. 39(3)(2010), p. 331. 